\title{
Analyser les mutations des espaces et des temps à l'ère de la digitalisation
}

Introduction au dossier « Espaces et temps de l'activité à l'ère de la digitalisation »

\section{Pascal Ughetto, Gaëtan Bourmaud et Yvon Haradji}

\section{(QpenEdition}

\section{Journals}

Édition électronique

URL : https://journals.openedition.org/activites/6459

DOI : 10.4000/activites.6459

ISSN : $1765-2723$

Éditeur

ARPACT - Association Recherches et Pratiques sur les ACTivités

Référence électronique

Pascal Ughetto, Gaëtan Bourmaud et Yvon Haradji, « Analyser les mutations des espaces et

des temps à l'ère de la digitalisation », Activités [En ligne], 18-2 | 2021, mis en ligne le 15 octobre 2021 consulté le 07 avril 2022. URL : http://journals.openedition.org/activites/6459 ; DOI : https://doi.org/ 10.4000/activites. 6459

Ce document a été généré automatiquement le 7 avril 2022.

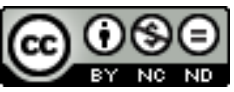

Activités est mis à disposition selon les termes de la licence Creative Commons Attribution - Pas d'Utilisation Commerciale - Pas de Modification 4.0 International. 


\title{
Analyser les mutations des espaces et des temps à l'ère de la digitalisation
}

\author{
Introduction au dossier « Espaces et temps de l'activité à l'ère de
}

la digitalisation »

\author{
Pascal Ughetto, Gaëtan Bourmaud et Yvon Haradji
}

1 La digitalisation s'est imposée, dans le courant de la décennie 2010, comme un défi que les organisations - privées ou publiques et quelle que soit leur taille - prétendent reconnaitre et affronter. Entreprises, administrations, organismes de différentes natures se sont mis à parler d'une transformation digitale ou numérique qui faisait surgir l'enjeu de leur survie ou de leur adaptation. L'expression a signifié qu'elles devaient se montrer disposées à réviser de fond en comble leurs modes de fonctionnement, à revenir sur des principes d'organisation et de management jusqu'alors présentés comme impérieux, à reconsidérer les pratiques quotidiennes et les modes de relation ordinaires. Dans cette perspective, la digitalisation ainsi entendue a fait naître des enjeux relatifs aux espaces et aux temps : elle est apparue comme impliquant un remodelage ou une restructuration du rapport aux espaces entre lesquels les individus (particulièrement, les salariés) circulent et des temps au sein desquels ils inscrivent leur action.

2 L'activité est-elle présente dans les raisonnements et les argumentaires qui ont été développés à cette occasion et dans les pratiques qui se sont formées? Qu'apporte un regard attentif à l'activité ? Ces questions ne sont absolument pas triviales, car elles amènent potentiellement à se demander si les structures de raisonnement des acteurs de la «transformation digitale » se concilient aisément avec ce type de regard ou si les acteurs engagés dans ces opérations de changement envisagent l'activité, la reconnaissent ou l'ignorent. S'ils parlent parfois d'activité, il faudrait encore vérifier dans quel sens. Sans qu'il puisse nécessairement apporter de réponse sur tous ces points, c'est, en tout cas, l'enjeu de ce dossier, dans une revue telle qu'Activités, que de traiter sur la base de cette problématique ou de ce type de prise de recul de mutations 
par ailleurs maintes fois documentées et commentées. Les perspectives généralement adoptées peuvent, par exemple, se préoccuper de vérifier ce que recouvrent concrètement les transformations annoncées, s'efforcer d'évaluer si elles réalisent la promesse formulée, si elles répondent réellement à des défis de performance économique et de bien-être au travail, etc. (Benedetto-Meyer \& Boboc, 2019 ; Brasseur \& Biaz, 2018; Valenduc \& Vendramin, 2016) L'objectif du dossier qui suit et des contributions qu'il réunit est, pour sa part, de se saisir de mutations en cours en ouvrant la question rarement soulevée à leur sujet de leur rapport à l'activité. La digitalisation a suscité un flot de commentaires et d'analyses dans lequel les propos très généraux et souvent spéculatifs l'emportent sur l'effort pour ancrer ces énoncés dans la référence à des situations et des activités précises, envisager la variabilité que la digitalisation risque de rencontrer ou préciser comment des principes alternatifs de fonctionnement et de management pourraient se matérialiser dans l'activité des personnels, y compris celle des membres de l'encadrement (Ughetto, 2018).

\section{Étudier la digitalisation à la lumière des usages des technologies et de l'activité}

3 Les discours sur la transformation digitale ont rapidement porté l'accent sur la nécessité de ne pas la réduire à la diffusion de technologies nouvelles - objets connectés, outils mobilisant l'intelligence artificielle, applications sur smartphones ou tablettes, etc. - et, au contraire, d'envisager que la transformation, réputée indispensable, devait avant tout être comprise comme renvoyant à des évolutions de comportement et de fonctionnement (souvent résumées en invoquant la nécessité d'une «transformation culturelle »). En ce sens, ils ne sont donc pas systématiquement portés au déterminisme technologique qui les menace pourtant a priori lorsqu'ils affirment que nous sommes confrontés à une mutation de grande ampleur dont l'origine tient d'abord dans l'essor des technologies numériques. Toutefois, ces discours ne mobilisent pas jusqu'au bout les repères conceptuels précisément forgés dans des décennies successives de débats en sciences sociales sur les effets des changements technologiques. Quels que soient les termes utilisés, ces débats ont appris aux spécialistes des sciences sociales à reconnaître l'importance de distinguer les propriétés de la technologie et les usages. Une technologie présente, certes, une structure qui, d'un côté, rend des actions possibles, augmente la puissance que recelait, sur tel ou tel registre, l'action humaine ou lui rend plus facile d'exécuter telle tâche, et, de l'autre, contraint cette même action, la force à respecter des scripts plus ou moins prescriptifs (Akrich, 1987). Mais la description de ces propriétés est loin de tout dire quant aux effets de cette technologie, tant que l'on ne s'est pas inquiété des usages effectifs : ce que les individus s'efforcent de faire, les significations qu'ils associent aux enjeux et aux situations, les utilisations non prévues par les concepteurs, les façons de procéder inventives, etc. Autant de rationalités qui s'expriment de façon autonome vis-à-vis des scripts de la technologie.

4 Sans doute la prise en compte de l'activité exige-t-elle une approche de la digitalisation susceptible de la voir comme une transformation - dont l'enquête devra déterminer l'effectivité et les formes réellement adoptées - posant la question des usages en train de s'ouvrir, afin d'interpréter la diffusion et de l'application des technologies concernées, les trajectoires suivies et les recompositions qu'on y observe. Raisonner en 
termes d'activité conduit à inscrire ces usages dans des contextes qui ne sont absolument pas anodins : les productions précises dans lesquelles les technologies sont mobilisées, les produits visés, le type de travail engagé. L'activité incite particulièrement à souligner que la digitalisation ne se manifeste pas en général ou dans l'abstrait, mais doit immédiatement être ancrée dans la spécificité des enjeux et contraintes d'un travail d'aide à domicile ou d'employé, de conduite de projet ou de traitement de dossiers, de réalisation d'un chantier du bâtiment, etc. Quels sont, en général, c'est-à-dire avant même la transformation digitale, les contraintes, ressources et enjeux de chacune de ces activités et quel sens prend alors l'offre technologique, quels usages cela s'avère-t-il susciter, de façon fréquente ou au contraire très variable ? Comment le travail recourt-il aux environnements socio-matériels - et donc notamment aux espaces - pour se réaliser, comment les pratiques agencent-elles les espaces et les temps à disposition afin que le travail se fasse?

\section{Digitalisation, espaces et temps dans les réflexions contemporaines sur les organisations du travail}

5 La digitalisation s'est trouvée associée à une réflexion sur les organisations du travail et, dans ce cadre, à des questions d'espaces et de temps de travail. Sur ce sujet, les acteurs ont souvent eu l'impression de se trouver face à des réflexions inédites, d'entrer dans une ère totalement nouvelle ouvrant sur des questions inexplorées. Les spécialistes des sciences sociales sont plus circonspects, au risque, inverse, de n'y voir qu'une simple progression des mutations technologiques amorcées avec ce qui était appelé, dans les années 1980 ou 1990, les « nouvelles technologies de l'information et de la communication ", avant qu'on ne parle de numérique (Benedetto-Meyer \& Boboc, 2021) : les effets sur le travail, les enjeux d'activité et d'organisation seraient alors pour partie ceux qui avaient déjà pu être mis en évidence lors des vagues de déploiement des logiciels de gestion intégrés, de la messagerie électronique ou dans d'autres encore, et pour une autre partie à analyser dans leurs manifestations plus inédites, comme celles liées à l'intelligence artificielle (Zouinar, 2020). En dépit du vague d'expressions telles que «transformation culturelle» dont ils se contentent, il ne faut pas ignorer ce mouvement des acteurs eux-mêmes, portés à ouvrir un débat sur les organisations du travail que les entreprises et les administrations ne supportaient pas d'avoir il y a encore quelques années. On assiste à la fois au prolongement de questions de recherche relatives aux liens entre mutations technologiques et travail et à l'ouverture de questions plus nouvelles autour de l'évolution des modes de fonctionnement et de management.

Derrière l'emprise du vocabulaire issu du marketing, qui a soudainement amené les organisations à s'enticher du terme "digital» au détriment de celui de numérique (Ughetto, 2018), se dessine la conviction des acteurs que les changements technologiques du moment véhiculent des mutations d'ampleur plus large pour leur propre fonctionnement. De même, l'engouement pour le terme de transformation, alors même qu'il pourrait parfois désigner de banales problématiques de conduite du changement, trahit la conviction, portée par les dirigeants d'entreprises, que le plus fondamental se joue dans la révision des fonctionnements hiérarchiques et dans la gestion des interactions qui permet aux salariés, individuellement et collectivement, de 
contribuer à des enjeux de «collaboration » et d'émergence d'idées, de produits et de procédés innovateurs.

7 C'est dans ce cadre que les espaces, particulièrement présents dans ce dossier, sont investis d'attentes. Les dirigeants ne les regardent plus exclusivement sous l'angle logistique et à partir des calculs de coûts qu'ils représentent, mais aussi comme des candidats à la performation de la transformation culturelle qu'ils appellent de leurs vœux: un moyen de la faire entrer dans les faits et en particulier dans les comportements des salariés. Le mot comportement s'entend bien dans le sens qu'il peut avoir en entreprise où une question permanente porte sur la possibilité d'envisager des conduites sociales ou actions humaines (celles des salariés ou, en marketing, celles des clients), caractérisées par ce qu'elles ont d'imprévisible, dans un format qui les rend scientifiquement explicables, répondant à des lois objectivables et par conséquent susceptibles d'être intégrées dans des systèmes de calcul et d'anticipation ${ }^{1}$. Cette perspective est-elle compatible avec une prise en compte de l'activité ? Le dossier permettra d'ouvrir cette question en se demandant si l'occasion se trouve ainsi donnée à l'activité de faire valoir ses impératifs et ses contraintes, notamment parce que la réfection des espaces de travail sous la forme de la mise en place de ce qui est généralement appelé le "flex office » emprunte beaucoup à une conceptualisation en architecture qui prétend concevoir des espaces fondés sur l'activité (activity-based working). On entend par là le fait que les architectes et aménageurs de bureaux ne penseraient plus les environnements de travail comme des postes attribués aux individus, mais comme devant répondre à des finalités du travail et à des activités de diverses natures (se concentrer sur un dossier, échanger avec des collègues sur un projet, converser par téléphone, participer à une réunion) chacune appelant, quel que soit le poste ou l'individu, des espaces adaptés. Dans la réflexion managériale, cela va de pair avec l'idée de promouvoir des «nouvelles façons de travailler » (new ways of working), incarnation de la transformation culturelle (Jemine, Dubois, \& Pichault, 2020 ; Taskin, Parmentier, \& Stinglhamber, 2019).

\section{Aborder les espaces et le temps en termes d'activité : les organisations y sont-elles prêtes?}

8 Avec le numérique, la technologie se trouve dotée de propriétés qui permettent de plus en plus aisément de desserrer l'étroite imbrication - remontant aux premiers temps de l'ère industrielle (Thompson, 1979) et surtout à l'émergence de la grande entreprise moderne à la fin du XIX ${ }^{\mathrm{e}}$ siècle (Noiriel, 1986) - entre l'attribution de tâches aux salariés par l'employeur, la désignation d'espaces pour se consacrer à ces tâches dans les murs de l'entreprise et la fixation de temps. En nouant étroitement tâches, espaces et temps, les employeurs se donnaient des moyens de contrôle sur le déploiement de l'activité, même s'ils ne parvenaient pas à assurer un recouvrement définitif entre tâche et activité.

9 Les discours sur la transformation digitale proclament que ce desserrement ne pose pas problème aux managers, voire se trouve appelé de leurs vœux, qu'il s'agisse de précipiter l'évolution des comportements, de répondre à des attentes prêtées aux salariés et notamment aux jeunes générations ou d'économiser des mètres carrés. Les organisations ouvrent, toutefois, ce faisant, la boîte de Pandore de l'activité et tout ce que représente celle-ci du point de vue du contrôle. Si l'activité ne se réduit pas à 
désigner une panoplie d'occupations (se concentrer, se réunir, chercher des idées dans une confrontation d'idées...), mais exprime le fait que s'acquitter d'une tâche revient inévitablement à prendre des libertés avec la prescription, le relâchement du lien entre tâche, espace et temps devrait faire de l'activité un sujet sensible. Si l'activité est constamment refoulée par les organisations, c'est que, quelles qu'elles soient - privées, publiques, associatives - ces organisations assurent la fluidité de leur fonctionnement et, au minimum, une performance productive en rationalisant les réponses qu'elles apportent à des problèmes auxquels elles se heurtent de façon récurrente. Elles formalisent, figent, homogénéisent ces réponses, moyen pour elle de rendre calculables (Vatin, 2008) et maîtrisables les innombrables actions et paramètres qui constituent leur fonctionnement et de s'assurer que ce qu'elles entendent produire comme biens ou comme services se fera en temps et en heure et dans le respect d'obligations réglementaires ou légales, de préoccupations de maîtrise des coûts, d'obtention éventuelle d'une rentabilité, etc. L'activité, les usages, se prêtent beaucoup moins facilement à cette calculabilité que la tâche et le « comportement » (réputé répondre à des lois générales). La tâche permet de procéder à des calculs sur la base desquels il est possible d'estimer les probabilités de tenir les objectifs quantitatifs ou qualitatifs.

Espaces et temps constituent des instruments au service du souci de ces structures de se maintenir dans les rails des objectifs qu'elles se donnent, objectifs qui les conduisent à ne pas être indifférentes aux chemins que se donne l'activité dans un certain écart avec la tâche. Relâcher la maîtrise du temps et des espaces et, à travers eux, celle de l'activité relève donc d'un pari que les perspectives en termes d'activité appellent de leurs vœux en maintes occasions, mais qui représente un vrai défi pour la gestion des organisations. Ce défi justifie que des recherches observent ces processus avec attention. Jusqu'à quel point des organisations peuvent-elles, à la fois, tenir leurs fonctionnements sous contrôle de leurs objectifs et laisser l'activité profiter de plus grandes latitudes en termes d'espaces et de temps pour emprunter des chemins plus inattendus? La conception même des espaces et l'organisation des temps n'intègrentelles pas la préoccupation d'octroyer des champs de liberté et un effort pour tenir sous contrôle certains de leurs usages possibles?

11 La crise pandémique liée au Covid-19 a amplifié les débats qui étaient déjà en cours quant aux possibilités croissantes d'effectuer le travail en dehors de l'espace et du temps normalement dévolus au travail. Elle ne fait sans doute qu'amplifier des processus marqués par l'enchevêtrement, au sein des espaces et des temps (murs de l'entreprise, transports, espaces domestiques, espaces de coworking...), d'activités personnelles et professionnelles (Célérier \& Monchatre, 2020). L'essor du télétravail réactive ces réflexions qui s'observaient, par ailleurs, dans les tentatives pour maîtriser les risques de sur-connexion. Dans chacun des cas, cependant, les discours des acteurs, les hypothèses à l'œuvre, les projections sont rarement ancrés dans la connaissance de l'activité. Le cadre de référence est souvent le comportemental, pointant les dérives auxquels les individus seraient portés et l'éducation à une meilleure hygiène de vie qui serait à leur proposer: se désintoxiquer de la consultation permanente de son smartphone et de ses e-mails, apprendre à séparer chez soi les temps et les espaces du travail et ceux consacrés aux impératifs domestiques, etc. (Créno \& Cahour, 2016; Greenan, Sardas, \& Ughetto, 2020 ; Prost \& Zouinar, 2015)

12 Le besoin est sans doute manifeste d'une attention à l'activité et d'une connaissance de celle-ci, afin de factualiser la discussion et d'outiller plus efficacement le dialogue 
social. Pourtant, la capacité des organisations de maintenir un tel cap est à étudier de près. Les organisations peuvent-elles apprendre à façonner de nouveaux agencements entre les dispositifs qui homogénéisent les fonctionnements pour tenir les objectifs et un plus grand pouvoir laissé à l'expression des logiques d'activité et à la reconnaissance de la variabilité à laquelle l'activité tente de répondre? Les dirigeants, les gestionnaires, les membres de la hiérarchie disposent-ils des outils conceptuels, dans la langue managériale, pour faire avec l'observation du cheminement de l'activité ? Les réflexions sur le comportemental, si elles ressurgissent dans de tels projets, ne sontelles pas l'indice d'une difficulté à doter d'un statut positif cette observation des faits relevant de l'activité?

\section{Les espaces de travail : quelle représentation de l'activité ?}

13 Issu d'une journée d'études organisée par Activités le 16 mai 2019 au Conservatoire national des Arts et Métiers (Paris), le dossier se penche sur les temps et espaces de travail, en les interrogeant à partir de la place qu'ils accordent à la prise en compte de l'activité dans un contexte de digitalisation où l'introduction du numérique va souvent de pair ou a pu précéder des refontes d'espaces de travail. Celles-ci se font désormais fréquemment sous l'égide des principes du flex-office où l'environnement de travail est supposé s'ajuster aux activités réalisées, sans évincer totalement la formule plus classique de l'open space. C'est également un contexte dans lequel les temps personnels et professionnels s'entremêlent de façon inextricable, de nouveau par l'entremise des outils numériques, et amènent les acteurs à rechercher les moyens de s'emparer de ces outils de façon à maîtriser les tendances à la sur-connexion.

Quatre articles constituent le dossier. Dans celui qu'ils intitulent «Penser l'appropriation des espaces de travail "par activités" par la mobilisation de la cognition située ", Chiara Lai, Maria Ianeva, Marc-Éric Bobillier Chaumon et Audrey Abitan nous font pénétrer dans les espaces réaménagés selon le principe du flex office. Le principe de leur conception est précisément qu'ils sont supposés s'adapter avec flexibilité aux activités de différentes natures auxquelles les individus se livrent dans le cadre de leur travail. Le travail d'observation et d'entretien conduit au sein de la filiale d'un grand groupe a été conduit en s'interrogeant sur les dynamiques d'appropriation de l'espace à partir d'une conceptualisation qui emprunte en particulier aux théories de l'action située et de la cognition distribuée. L'espace de travail participe entièrement du besoin, dans la conduite de l'activité, de se doter de ressources pour agir, de se forger des instruments, et cela de façon située, directement ancrée dans un contexte sociomatériel qui, tout à la fois, contraint et équipe. Inévitablement, dès lors, les individus et les collectifs façonnent l'espace pour lui donner valeur d'instrument. C'est ainsi, montre l'article, que l'insertion dans les espaces réaménagés ne se borne pas à l'utiliser, dans un sens relativement mécanique et attendu, mais consiste à confectionner des règles de bon usage des différents lieux, à remodeler dans un même mouvement lieux et pratiques, à accommoder les nouveaux espaces autant qu'à s'accommoder à eux.

Dans «Espace, travail et numérique : le cas du travail en flex office ", Nadia Heddad adopte un point très proche et présente des conclusions fortement convergentes, appuyées par une étude de cas dans les services du siège d'un autre grand groupe. Influencée par la distinction qu'avait proposée Henri Lefebvre à propos des villes entre 
l'espace conçu, l'espace perçu et l'espace vécu, elle s'attache à souligner toute la différence qui ne manque pas de s'exprimer entre l'espace conçu pour la tâche et celui qui se révèle modelé et agi par l'activité de travail. Les outils numériques, qui n'avaient pas attendu le flex-office pour se déployer, mais que celui-ci appelle encore plus fortement, ajoutent cependant des effets flagrants de porosité spatio-temporelle dans et hors de l'entreprise. La décorrélation entre la production et les lieux se trouve amplifiée par la conjonction entre flex-office et outils numériques. L'activité réelle des personnels tend à être invisibilisée. Mais également les méthodes d'analyse de l'activité devront sans doute évoluer pour s'adapter à ces contextes doublement marqués par ces bureaux de nouvelle génération et le numérique.

La gestion de cette porosité des espaces et des temps est au fondement de l'article signé par Vincent Grosjean, Ophélie Morand, Béatrice Cahour et Marc-Éric Bobillier Chaumon, «E-conciliation vie de travail / vie hors-travail : intervenir par le théâtreforum ». Le problème posé par la sur-connexion a été maintes fois identifié et l'effort des acteurs pour parvenir à la réguler voire à favoriser la déconnexion est aussi constant que difficile à faire aboutir. C'est le mérite de l'article de ces quatre auteurs que de proposer une analyse précise de la mise en œuvre d'une méthode, celle du théâtre-forum, dont Vincent Grosjean, en particulier, est familier. Elle organise un espace d'échange dans des collectifs de travail pour aider les membres de ces collectifs à identifier les problèmes et, par la mise en scène et en débat, de cheminer vers la construction de solutions. Encore une fois, cet article permet de percevoir l'extrême imbrication entre normes implicites et usages et comment l'activité se trouve constamment intégrée dans les espaces et le temps, à la fois contraignants et remodelés. Aider à " conscientiser », disent les auteurs et autrices, est la condition pour que des collectifs puissent reprendre la main sur un problème complexe qui dépasse les capacités transformatrices de chacun des individus pris un par un.

17 Enfin, Nathalie Trisse et Christine Lagabrielle, dans « L'approche de l'acceptation située comme modèle de compréhension du processus d'appropriation des espaces de travail : le cas d'une collectivité locale aménagée en open space » nous font revenir vers une génération plus ancienne d'aménagement des environnements de travail, qui n'est cependant pas entièrement déclassée par le flex-office. Les grands plateaux en espace ouvert demeurent le cadre de travail de beaucoup de salariés, même à l'ère de la digitalisation. Les deux autrices partagent avec les autres publications du dossier la conviction de la nécessité d'une approche travaillant sur les processus d'appropriation pensés à partir du fait que l'activité est profondément située. Dans cet article qui porte particulièrement sur la modélisation pertinente de ces processus, une "clinique de l'usage » est revendiquée comme un instrument d'analyse indispensable de ces processus. Là encore, il faut une intervention, une médiation, pour aider les acteurs à faire avec ces espaces, à développer des appropriations où cessent de dominer les facteurs d'empêchement qui peuvent, dans un premier temps et spontanément, être prioritairement à l'esprit des acteurs concernés. Reconstruire un collectif, séance après séance, fait partie de ce qui va permettre d'investir plus positivement l'espace et d'y forger sa dimension d'instrument.

18 Ces articles convergent autour de principes d'analyse et de messages forts. Ils demandent aux lecteurs d'appréhender l'espace comme étant autant défini par la tâche que restructuré par l'activité et comme ne représentant pas une donnée objective et impersonnelle de l'environnement, mais comme se trouvant saisi par des 
interprétations et des pratiques de la part de sujets autonomes ou d'acteurs qui agissent de façon située : les espaces participent de la situation de l'action, ils ne se limitent pas à un simple décor. Ils fournissent - plus ou moins - des appuis de l'action et sont reconstruits dans l'action afin de fournir des instruments à l'activité. Même, répétons-le, si les espaces sont investis par les managers d'un enjeu de performation de la transformation culturelle, ils sont inévitablement l'objet de processus d'appropriation qui, comme a pu le montrer Marc-Éric Bobillier Chaumon (2016) pour les technologies (dans une problématique que plusieurs auteurs ici adaptent aux espaces) doit être comprise comme une acceptation située : l'appropriation renvoie aux pratiques qui, pour se déployer, s'ancrent dans le contexte, sont contraints par lui, mais y cherchent également des points d'appui.

Dans le cadre de ces processus d'appropriation se développent des usages. Ceux-ci débordent largement du chenal que les concepteurs des espaces envisageaient et les acteurs s'emploient à les réguler. Aussi, les controverses autour des normes morales, des comportements légitimes, des principes nécessaires sont constamment au cœur de l'élaboration des nouveaux usages. Cette place est identique dans les pratiques de connexion, d'utilisation d'outils numériques qui permettent l'intrusion du domestique dans des temps professionnels collectifs et inversement, et les dynamiques qui permettent à des collectifs d'apprendre à en débattre et à élaborer des régulations discutées se révèlent cruciales dans ces processus de transformation.

Une fois de plus, ce dossier prétend davantage montrer la nécessité d'interroger les mutations en cours à partir d'un prisme exigeant, celui de l'activité, qu'apporter des résultats définitifs. Il constitue une invitation à développer de nombreux travaux autour de cette perspective dans les années à venir. Souhaitons-lui ce destin.

\section{BIBLIOGRAPHIE}

Akrich, M. (1987). Comment décrire les objets techniques ? Techniques et culture, 9, 49-64.

Benedetto-Meyer, M. \& Boboc, A. (2019). Accompagner la «transformation digitale » : du flou des discours à la réalité des mises en œuvre. Travail et emploi (159), 93-118.

Benedetto-Meyer, M., \& Boboc, A. (2021). Sociologie du numérique au travail. Paris : Armand Colin.

Bobillier Chaumon, M.-E. (2016). L'acceptation située des technologies dans et par l'activité : premiers étayages pour une clinique de l'usage. Psychologie du Travail et des Organisations, 22(1), 4-21.

Brasseur, M., \& Biaz, F. (2018). L'impact de la digitalisation des organisations sur le rapport au travail : entre aliénation et émancipation. Question(s) de management, (21), 143-155.

Célérier, S., \& Monchatre, S. (2020). Fragmentations du travail, continuité productive et épreuves du temps. Temporalités, (31-32).

Créno, L., \& Cahour, B. (2016). Les cadres surchargés par leurs emails : déploiement de l'activité et expérience vécue. Activités, 13(1). https://journals.openedition.org/activites/2698 
Greenan, N., Sardas, J. C., \& Ughetto, P. (2020). Organiser la déconnexion? Travail intense et articulation des temps et des espaces dans les activités de conception d'un groupe automobile. Temporalités, (31-32).

Jemine, G., Dubois, C., \& Pichault, F. (2020). When the gallic village strikes back: The politics behind 'New ways of working'projects. Journal of Change Management, 20(2), 146-170.

Noiriel, G. (1986). Les ouvriers dans la société française, XIX ${ }^{e}-X X^{e}$ siècle. Paris : Le Seuil (Points).

Prost, M., \& Zouinar, M. (2015). De l'hyper-connexion à la déconnexion : quand les entreprises tentent de réguler l'usage professionnel des e-mails. Perspectives interdisciplinaires sur le travail et la santé, 17(1).

Robbins, S., \& Judge, T. (2006). Comportements organisationnels. trad. fr. Pearson.

Taskin, L., Parmentier, M., \& Stinglhamber, F. (2019). The dark side of office designs: towards dehumanization. New Technology, Work and Employment, 34(3), 262-284.

Thompson, E.P. (1979). Temps, travail et capitalisme industriel. Libre (5), 3-64.

Ughetto, P. (2018). Organiser l'autonomie au travail. Travail collaboratif, entreprise libérée, mode agile : l'activité à l'ère de l'auto-organisation. Limoges : FYP.

Valenduc, G., \& Vendramin, P. (2016). Le travail dans l'économie digitale : continuités et ruptures. ETUI Working Papers, (2016.03), $56 \mathrm{p}$.

Vatin, F. (2008). L'esprit d'ingénieur : pensée calculatoire et éthique économique. Revue française de socio-économie, 1(1), 131-152.

Zouinar, M. (2020). Évolutions de l'intelligence artificielle : quels enjeux pour l'activité humaine et la relation Humain-Machine au travail ? Activités, 17(1), en ligne. https://

journals.openedition.org/activites/4941

\section{NOTES}

1. Une spécialité à part entière des sciences de gestion a fini par s'autonomiser, précisément intitulée comportements organisationnels (organizational behavior). Assez significativement, la discipline se présente elle-même en évitant soigneusement de définir son terme principal, quitte à opter pour une définition circulaire du comportement organisationnel comme celle de Robbins et Judge dans leur manuel ("Domaine d'étude visant à explorer l'impact des individus, des groupes et des structures sur le comportement des acteurs d'une organisation en vue d'améliorer son efficacité ", Robbins et Judge (2006, p. 10). Cette définition a au moins le mérite de laisser apparaître que la possibilité voire la nécessité que les comportements se montrent fonctionnels vis-à-vis des besoins d'efficacité de l'organisation sont au cœur de l'épistémologie adoptée. On pourra donc proposer à ce champ scientifique de définir le comportement, son objet, comme étant une mise en forme des conduites sociales et actions humaines permettant de les rendre a) passibles d'une analyse scientifique positiviste dégageant des lois, b) interprétables dans la perspective des objectifs d'organisation d'une entreprise ou d'un autre type de structure et c) rendues suffisamment calculables pour aider à la prise de décision et à la formulation de règles d'organisation. 